\title{
Large-Scale Distributed Computational Fluid Dynamics on the Information Power Grid using Globus
}

\author{
S. Bamard; R. Biswas;" S. Saini, R. Van der Wijngaart;" M. Yarrow! L. Zechtzer* \\ NAS Division, NASA Ames Research Center, Moffett Field, CA 94035, U.S.A. \\ \{barnard,rbiswas,saini,wijngaar,yarrow,lou\}@nas.nasa.gov \\ I. Foster, O. Larsson \\ MCS Division, Argonne National Laboratory, Argonne, IL 60439, U.S.A. \\ \{foster,larsson\}@mcs.anl.gov
}

\begin{abstract}
This paper describes an experiment in which a largescule scientitic application developed for tightly-coupled parillel machines is adapted to the distributed execution environment of the Information Power Grid (IPG). A brief oicriew of the IPG and a description of the computational fluid dynamics (CFD) algorithm are given. The Globus metacomputing toolkit is used as the enabling device for the geographically-distributed computation. Modifications related to latency hiding and load balancing were required for an efficient implementation of the CFD application in the IPG environment. Performance results on a pair of SGI Origin2000 machines indicate that real scientific applications can be effectively implemented on the IPG; however. a significant amount of continued effort is required 10 make such an environment useful and accessible to scientists and engineers.
\end{abstract}

\section{Introduction}

In one of its Enabling Technologies Goals. NASA has committed to "provide next-generation design tools and experimental aircraft to increase design confidence and cut the development cycle for aircraft in half." To meet such ambitious goals. a significant improvement is required in NAS.A's ahility to create, process, understand, store, and communicate data. It is unlikely that conventional approaches to high-pertormance computing will he able to acheve these loity nhjectives. Therefore. NASA is building a nationwide infrastructure called the Infomution Power Grid (IPG).

\footnotetext{
- Iinplnyee of MRJ Tecthnology Sulutiuns.

timployce of Sicerling Suftwals:
}

The IPG is intended to provide ubiquitous and uniform access, through a convenient interface, to a wide range of computational, communication, data analysis, and storage resources, many of which are specialized and cannot he replicated at all user sites. It imvolves linking the vast. heterogeneous, and geographically-distributed resources of NASA and its IPG parners to create a scalable, adaptive, and transparent computational environment. The interface will hide details of machine particulars, such as location, size, connectivity, and name, thereby presenting users with a unified virtual machine. A blueprint for this proposed technology is documented in [6].

The IPG can be used to address two major computing requirements. The first involves efficiently orchestrating several technologies to enable distributed human collaboration and location-independent access to unique resources. The goal is to create an integrated environment that allows researchers to solve specific problems quickly. The second requirement is to provide a transparent, widelydistributed. high-performance metacomputing facility to solve extremely large applications that are currently intractable on rightly-integrated paral lel supercomputers.

The goal of the work reported in this paper is a proof-ofconcept demonstration of how IPG technology can be used effectively to tackle a challenging problem in this second area: runuing a single, large compurational fluid dynamics (CFD) application in a distributed fashion on separate machines. The virtual distributed computer is viewed as a collection of supernodes, where each supenode is a homogeneous, tightly-coupled machine.

The Globus project [5] aims to develop a sofiware inirastnucture for computations that integrate geographicallydistributed computational and information resources. At present. it is a readistic staning point for the implenuentilion of lle IPG. Glohus has already heen deploved on a 
large testbed, called GUSTO, spanuing 40 sites and providing 2.5 Thlops of compute power. In this paper, we describe how Globus is used to combine homogeneous but distributed resources (SGI Origin2000 systems at NASA Ames Research Center and at Argonne National Laboratory) to simulate an X-38 Crew Return Vehicle (CRV). This experiment provided experience with Globus and insights into the requirements for future IPG technology at NASA.

The CFD application chosen for this experiment involves the accurate prediction of high-speed viscous flow around a geometrically-complex three-dimensional body. Problems of this nature challenge the capabilities of the most advanced single-processor platforms available. Large-scale multiprocessor computer systems offer a powerful tool to solve large and complex problems; but they may still not suffice, and gaining exclusive access to them is difficult in practice. The CFD software used is an enhanced version of OVERFLOW [2], the most widely-used flow solver software at NASA Ames. OVERFLOW deals with the geometrical complexity of flow solution domains by allowing sets of separately generated and updated structured discretization grids to exchange information through interpolation.

The main technical challenge in implementing scientific applications on the IPG lies in accommodating the sizable and variable latencies as well as the reduced bandwidths incurred in distributed computations on geographicallyseparated machines. Common latency-hiding techniques such as pipelining can only be used when data dependencies are known in advance and when data can be prepared and sent long before it is needed. For example, real-time visualization of scientific data can be formulated as a two-stage pipeline, with one machine generating the data and the other performing the rendering. But such strategies are useless for running tightly-coupled applications like OVERFLOW in a distributed manner, where computation and communication are intrinsically interleaved. The focus of this work is on identifying and implementing the minimum changes to a state-of-the-art parallel program that are necessary to run it efficiently as a distributed application in an IPG environment.

The remainder of this paper includes a brief overview of the IPG concept and the enabling software layer (Globus) used in this project (Sec. 2), a description of the CFD scheme and an outline of the application used for our experiments (Sec. 3), a description of the IPG implementation of the application and computational results (Sec. 4), and summary remarks and a discussion of future directions (Sec. 5).

\section{Distributed computing environment}

Onc of the early success stories in the history of distrihuted computing hats teen the Parallel Vinual Maclunte (PVM) |7| library. However, the subseçuent substantial use of PVM al so revealed some of the limitations of ure concept of the library. Much of the burden of using it lor distributed computing fell on the user. For example:

- All remote computer resources had to be named within the application program.

- All information needed for creating and running remote processes (executables, data files, scripts. etc.) had to be moved to the proper location by the user.

- All remote processor and file system access issues, most notably security and accounting, had to be explicitly resolved by the user.

This led to the realization that a more top-down approach to distributed computing is needed, in which an integrated environment for distributed applications and remote services is provided without requiring an undue amount of effort on the part of individual users. This quest for an integrated but distributed environment forms a substantial pillar of the IPG project.

\subsection{Information Power Grid}

The Information Power Grid (IPG) project is being conducted by NASA in collaboration with a number of govelrument and academic parners. A large number of compute and data resources are currently available in principle to NASA researchers, but they are often not easily accessible from different locations. The goal of the IPG is to make these resources available easily, uniformly, and transparently. The IPG is intended to facilitate the aggregation of these distributed resources to enable scalable systems required to solve problems that are intractable on current localized computing environments [10]. More specifically, the IPG is meant to support aerospace research and engineering, with their typical requirements of large-scale simulations and very data- and compute-intensive visualizations.

A layered design of IPG, depicted in Fig. 1, makes the technical implementation manageable. The bottom two layers constitute the existing NASA computing environment, and consist of the native operating system and the basic hardware. The top layer, called Aerospace Engineering System, provides a number of tools and interfaces that are specific to future research in aerospace engineering. The Tools and High-Level Services layer provides supplemental tools and interfaces to make the IPG Virtual Machine more user-friendly and easier to administer and maintain. All these four layers are beyond the scope of this paper.

The long-term objectives of the research effort behind this paper is to investigate, develop, and demonstrate technology for the IPG Virtual Machine layer. This layer provides a uniform intertace for the user to specify computational or informational tasks, regardless of where the lasks are 10 the exceuted. It consists of five separate subsystems. as sllown in Fig. 1. This layer constitutes a vinual dis- 


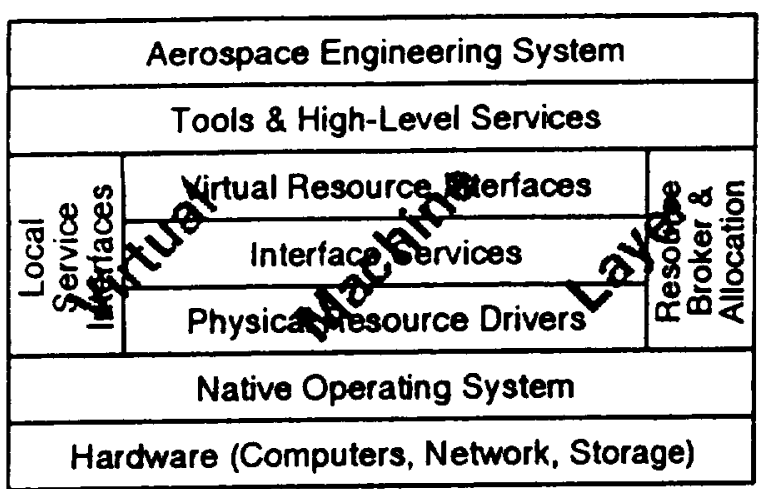

Figure 1. Layered structure of the Information Power Grid.

tributed computing resource that resolves issues of compatibility hetwcen the heterogeneous systems spread around the organizations of NASA and its partners. This requires tools for specifying memory requirements, the number of distributed processes, processing speeds, compilers, wall clock time nceds. libraries, header files, and so on.

\subsection{Globus metacomputing toolkit}

Several recent research projects $[1,5,8,15]$ provide prototypes for the IPG Virtual Machine layer. In this paper we test one of them, the Globus metacomputing toolkit [5], as a possible realization. Globus evolved out of the successful but still somewhat ad hoc I-WAY high-performance distributed computing experiment [4].

Globus provides core services required for the IPG Virtual Machine, including the management of resource location and allocation, communication, gathering unified resource information, authentication, remote process creation, fault detection, and remote data access. For the purpose of the experiments reported in this paper, in which dedicated resources are used, the critical Globus components are communicationi and process creation. Communication is implemented throtigh MPICH-G [3], a Globus-enabled device firs the public-domain implementation of MPI [9]. and process creation through the co-allocation service accessed via the globusrun utility used to interface with local schedulers. While Globus is used to make the two remole parallel computers recognize each other, the entire application is run as a single message-passing program under MPICH, and the application progranmer need not he aw:ure of any distinction between the two machines.

\section{Computational fluid dynamics application}

The CFD scheme used in this work utilizes strnctured oversel grids based on the Chimerid ||4| style of domain decomposition. Such schemes have proven to be appropriate for predicting high-speed viscous flows around complex shapes for both static and dynamic (i.e., moving-body) configurations. The Chimera scheme divides the entire problem domain into a system of grids that overlap one another by one or more grid cells. The solution proceeds by updating, at each iteration, the inter-grid boundaries on each grid with interpolated data from overlapping grids. Geometrically-complex shapes are broken into groups of overlapping curvilinear body-fitted grids and relatively simple rectilinear background grids. Besides being numerically expedient, the domain decomposition nature of the Chimera approach offers a high degree of coarse-grained parallelism that can be exploited in distributed computing environments.

The most popular overset-grid flow solver that is used at NASA Ames Research Center is the OVERFLOW code [2]. There are at least three different parallel implementations of OVERFLOW, using different programming models and data-distribution methods. The first is a fine-grained MPI version where it is possible to partition individual grids among processors; however, it does not have all the various boundary conditions and grid types implemented. The second version is an MLP (multi-level programming) code designed specifically for the CC-NUMA architecture of the Origin2000. It is able to balarice the processor workloads automatically based on runtimes from the first few iterations, but does not work on multiple loosely-coupled machines.

The third version is the one used for the experiments reported in this paper. Here, each grid is assigned to a unique processor, and the set of grids assigned to any paricular processor is referred to as a group. In this coarse-grained data-distribution scheme, it is only necessary to communicate some of the boundary information between processors. The computation for individual grids is entirely serial.

A number of enhancements have been made within this third version of OVERFLOW that allow solution-based mesh adaptation [12] and scalable parallel execution [16]. MPI message passing facilitates execution on a variety of distributed computer platiorms, and the code has been successfully tested for static geometry problems on an IBM SP multiprocessor.

\subsection{Parallel implementation}

Overset grid schemes helong to the general class of Schwarz domain deconposition methods. Since each subdomain boundary is updated on y once per iteration with interpolattions trom neightoring subdomains possibly reguiring communications. a natural coarse-grained level of parallelism exists. The automated Cartesian grid generıtion scheme generites a relatively large number of grids, usuadly 
many more than the available number of processors. This trend is expected to continue as computational problems become larger and more complex.

Because there are many more grids than processors, our distribution approach places one or more grids onto each processor in a load-bal anced fashion. A grouping al gorithm seeks to balance the computational workload by considering both the number of grid points in each group and an estimate of the work associated with each grid point. The latter is necessary because some grids may require more computational work per grid point than those in others. For example, a turbulence model may be applied on grids near the body but not on grids in the far field. Weighting factors that correspond to the additional work are applied to the grids containing the more computationally intensive points, prior to performing the grouping. The grouping strategy also seeks to maintain a degree of locality among the member grids in each group to maximize the level of intra-group connectivity. Details of the bin-packing groupin', al gorithm are given in [16].
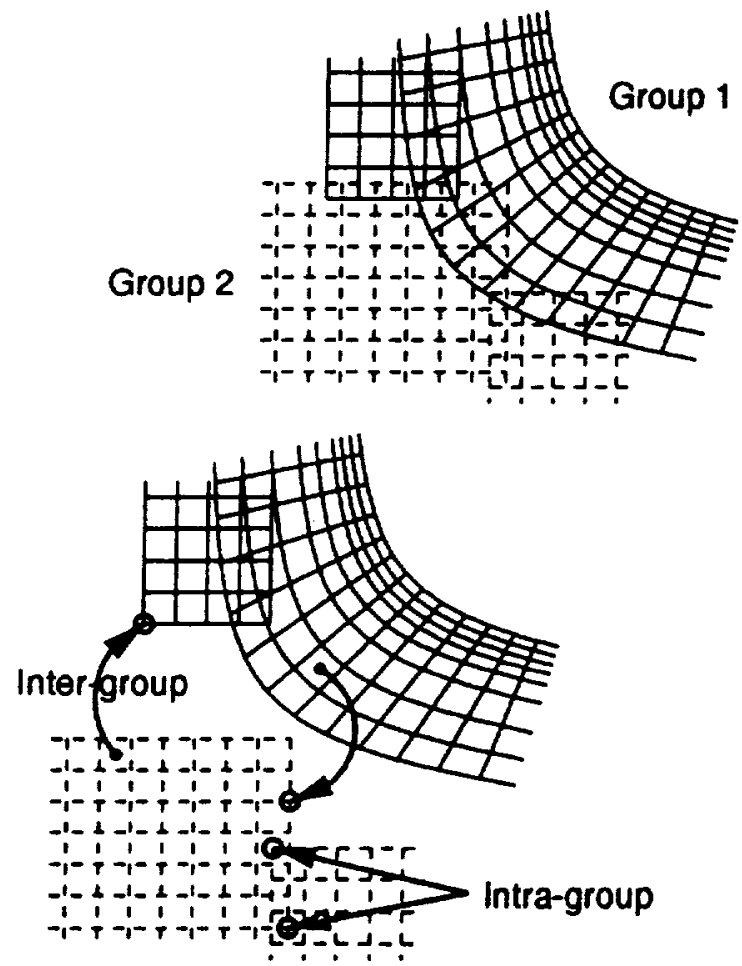

Figure 2. Intragroup and inter-group interpolations between grids.

Boundary information is interpolated between overlapping grids at cach iteration. Grids that are in the same group pertiorm intra-group interpolations locally on each processor. Grids that overlap with other grids in a different group pertorm intcr-group interpolations between processors. For the latter case, the donor value's supplied to the neighboring group are compuled locally and then exchanged using MPI calls. This approach is outlined schematically in Fig. 2 , where two groups are shown, each containing two grids. Both intra-group and inter-group interpolations take place at the end of each iteration; hence. interpolated data on all grids lag by one iteration.

The rectilinear-grid generator occasionally generates grids that are individually much larger than the average size of a group. This situation leads to either load imbalance or to grids that do not fit in core. To avoid this situation, the grids are checked after generation, and a recursive binary splitting technique [16] is applied to those grids that exceed the average group size. The rectilinear-grid generator then automatically generates the appropriate system of grid components and computes the necessary interpolation stencils. The spliting procedure is significantly more complicated for the body-fitted curvilinear grids and has not yet been implemented for such grids.

\subsection{Test case}

The parallel version of the overset CFD code has been implemented on the IBM SP at Army Corps of Engineers Waterways Experiments Station (CEWES). Test cases include steady-state viscous calculations of two relatively complex aerodynamic configurations: NASA's X-38 Crew Return Vehicle (CRV) and Army's Comanche helicopter (without blades). The former, shown in Fig. 3, is used for the IPG simulation experiments reported in this paper.

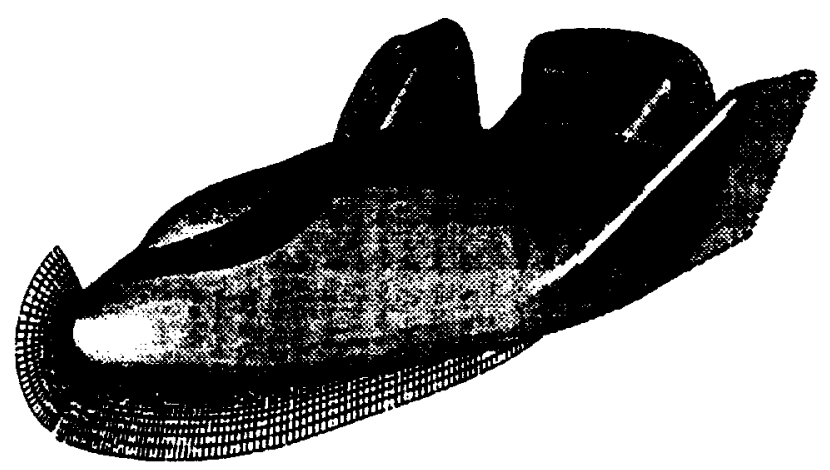

Figure 3. View of the X-38 Crew Return Vehicle.

The grid system for the X-38 CRV consists of 13 nearbody curvilinear grids and 115 off-body rectilinear grids, for a total of more than 2.5 million points. The largest grid is hody-fitted, and contains $4.37,976$ points. A single grid this large causes scalability problems; tuture work will address this isstec. The smidlest of the 128 overlapping grids contains only 216 points. Having many small grids is desir- 
able trom a load balancing perspective, but may harm the convergence of the numerical scheme.

Parallel pertiormance results on a single supercomputer are available in [16]. Runs on up to nine processors of the IBM SP show a total communication cost of only $2 \%$ of the entire calculation. The deterioration of the parallel efficiency to $88 \%$ when going from tour (the smallest number of processors required to do in-core computation) to nine processors is due mostly to a poorer load balance as the number of grids per processor decreases. This can be alleviated to some extent by breaking large grids into several sinaller ones.

\section{IPG implementation}

As mentioned in Sec. 3, the enhanced version of OVERFLOW has heen developed as a coarse-grained parallel program tor tightly-coupled parallel machines. The communication overhead on a fairly richly-connected architecture such as the IBM SP is typically about $10 \%$ of the total execuiton time on 128 processors. However, in a truly disinhi:ted IPG cnvironment with poorer connectivity (smaller ،ggregate baindwidth) and significantly larger latencies due to the geographical separation of the computers used, modifications must be made to reduce the impact of communication. This is achieved in two phases.

First and most notably, a higher level of asynchrony must be embedded into the numerical scheme to hide latency. This issue is addressed in Sec. 4.1. Second, a more sophisticated techuique must be used to map the overset grids to the supernodes of the IPG and, in turn, to the individual processors of these supernodes. This is described in Sec. 4.2.

\subsection{Latency hiding}

The time-advancement strategy of the solution scheme on the overlapping grids has been altered to hide the increased latency between IPG supemodes. In the original parallel scheme, all communicating processors first exchange thundary values. Once the exchange is completed; the interior solution domains are updated independently. Consequently, the entire communication is exposed, and ovcral! pertormance deteriorates as the connectivity degridis. In the new scheme, latency tolerance is obtained by lis:uing the boundary value update hy one additional limesicp. The houndary value exchange is initiated at the besinning of a timestep, but lhe values are not used until the heginning of lle nex timestep. It allows the overlap of computallion and communication lor as much as the duration of onc entice timestep. We call this the deferred scheme. Both llwe origriall and llke detierted schemes are depicled schentaticilly in Fig. 4.
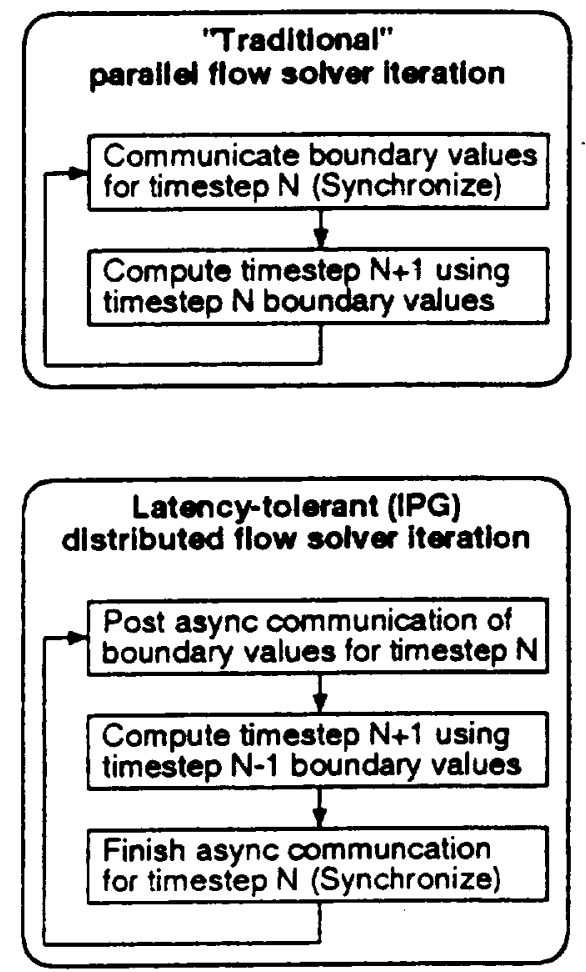

Figure 4. Creating flow solver latency tolerance for the IPG environment.

One potential problem with the deferred scheme is that lagging the boundary value updates may render the flow solver unstable or cause it to converge less rapidly. However, the results reported in Sec. 4.3 show negligible difference in convergence or in the values of some physical quantities for the X-38 CRV simulation (which is basically a steady-statc case except for some unsteadiness belund the vehicle that does not affect the solution at the leading edge or on the body).

The possibility remains that the deferred method may lead to instability or slower convergence for more difficult unsteady prublems. However, nore that it is only necessary to use the latency-tolerant method at the boundary between the supermodes, where the high latencies are encountered. It may be possible to partition the collection of grids between the supernodes so that the boundary encompasses only a relatively steady, slow-changing part of the solution, thereby avoiding instability or convergence problems.

\subsection{Load balancing}

The original method tor grouping grids and assigning uroups 10 processors described in Sec. 3.1 is ad hoc, hut quite cficective at ballancing load on a tightly-coupled parallel system lior moderate-sized prohlems (assuming that a 
good load balance is possible for a given grid system and number of processors). It mosuly ignores the cost of communication between grids, except to the extent that it attempis to place neighboring grids on the same processor: As long as the communication overthead is relatively insignificant, this will be effective. On a system consisting of two or more loosely-coupled supernodes, however, the greatly increased latency and reduced bandwidth between the supernodes can have a substancial impact. Moreover, since large numbers of processors will be required for future very large-scale computations, we are led to investigate a more principled method for load balancing.

The problem of assigning grids to processors in a way that balances load and minimizes communication is a classic partitioning problem, which often arises in distributed unstructured applications. Such a mapping mitigates the effect of reduced bandwidth. The set of grids defines an undirected graph. with the grids represented by the vertices and the overlap between pairs of grids represented by the edges. An estimate of the work required by each grid can be used to define a weight for each vertex, and an estimate of the cost of each inter-grid interpolation can be used to define a weight for each edge.

Thic patitioning problem is to assign $v$ vertices to $P$ processors $(P \leq v)$ such that each processor has a (roughly) equal aggregate vertex weight, while the total weight of the edges that span different processors is minimized. (Figure 5 shows a small example for $v=20$ and $P=4$ with uniform vertex weights and edge weights). This problem has been thoroughly studied. While it is NP-hard, several heuristic solutions are very effective. We have integrated one such partitioner, called MeTiS [11], into our flow solver code, and find that it distributes grids as effectively as the original scheme. This is because the X-38 CRV test case contains a few' very large grids that dominate the workload. It is therefore impossible to improve the quality of the load balance without splitting the large grids. However, the MeTiS partitioner will generally be more useful for larger problems with more grids.

Another weakness of the original load balancing method is that it estimates the work required by each grid from the number of grid points. (Near-body grids are treated slightly differently from off-body grids.) While the number of floating-point operations is proportional to the number of grid points, cache effects may introduce nonlinearities because the grid size may interact with the various cache sizes in unpredictable ways. A comparison of the estimated work and the actual work required for each of the 128 grids of the X-38 CRV revealed considerable discrepancies: as ligh at $1.3 \%$ tor one hody-litted grid and $35 \%$ for one oft-body grid. Instead of using mere estimates tor grid weights, we use actual meisurements. Using a default partitioning. the sinulition is nun for a tew timesteps and the work tor each

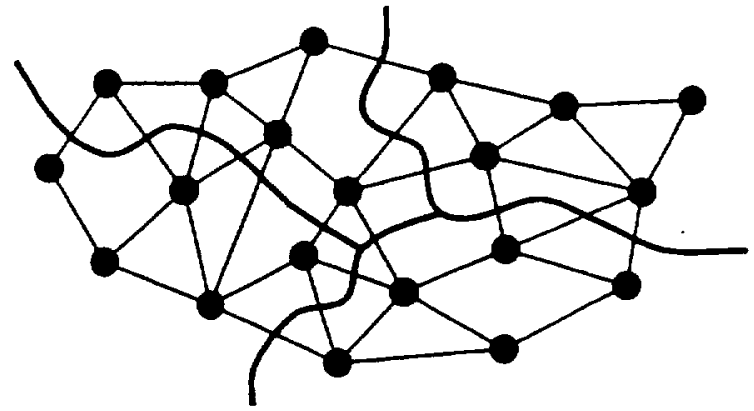

Figure 5. Partitioning an unstructured graph while balancing computation and minimizing communication.

grid is measured. These measurements are then used to produce a final partitioning.

\subsection{Results}

Our IPG testbed consisted of three separate SGI Origin2000 machines: two located at NASA Ames Research Center and the third at Argonne National Laboratory. A maximum of 8 processors were used on any one machine. each running Globus version 1.0.0. MPICH-G was used as the message-passing library.

The flight test conditions for the X-38 CRV were a Mach number of 1.5 and a 15-degree angle of attack. Figure 6 shows the computed Mach contours on the symmetry plane. Additional details of the simulation and computed loads are given in [12].

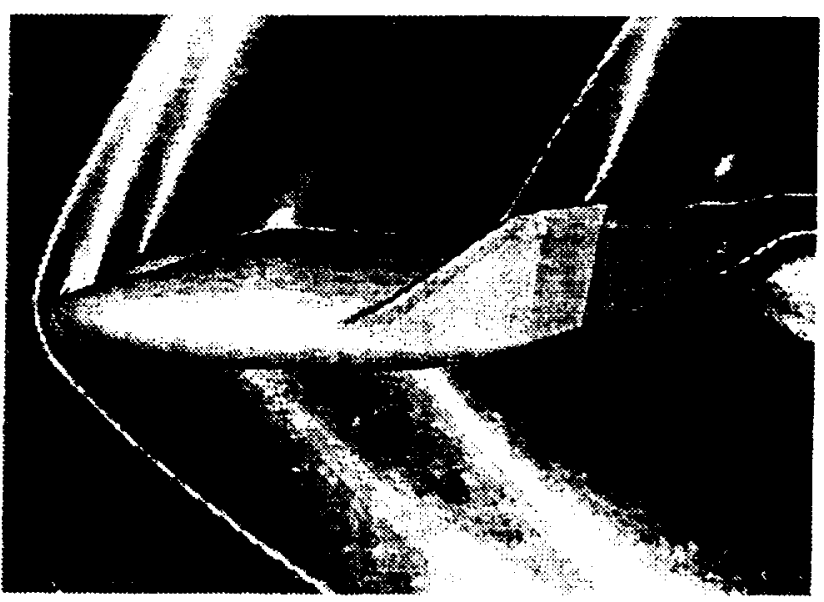

Figure 6. Mach contours for the X-38 Crew Return Vehicle.

The first experinemt wats to compare the results of computations using the original and delerred versions of the 
How solver (as described in Sec. 4.1 ) for the $X-38$ configuration. Figures 7 and 8 show the difference in the $L^{2}$-norms of the residuals of the iterative scheme for two representative near-body grids. Reduction of the residual on these grids is a good measure of the overall convergence of the method.

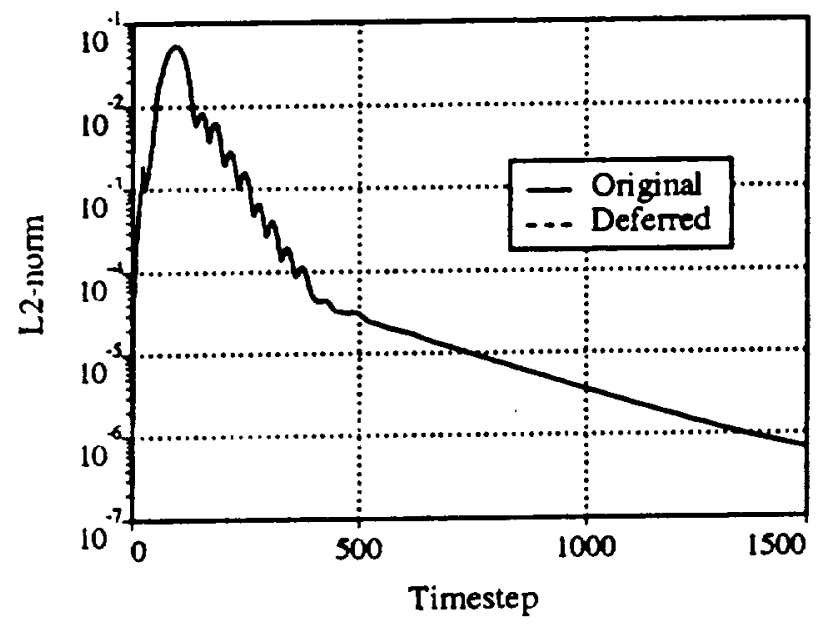

Figure 7. Residual comparison for the $X-38$ CRV nose cone (grid 1).

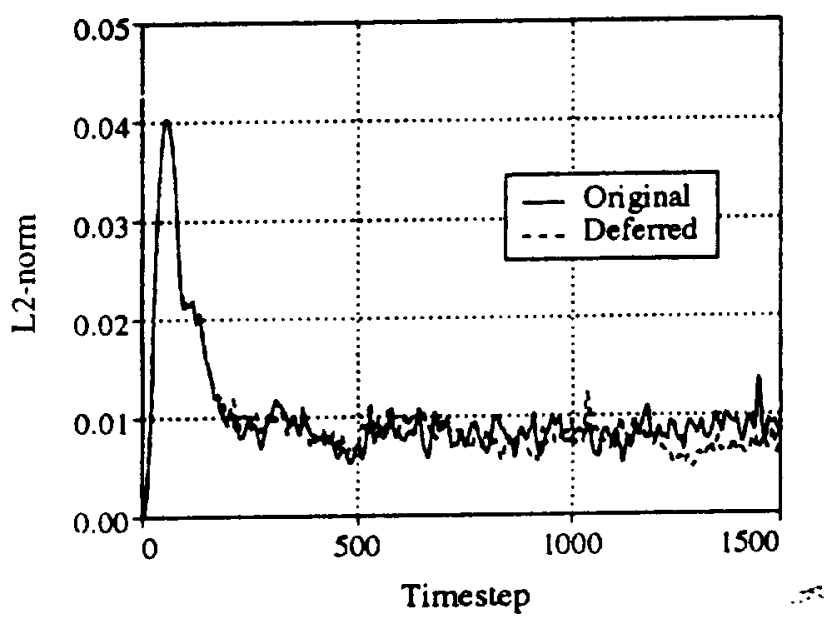

Figure 8. Residual comparison for the $X-38$ CRV rear section (grid 5).

Clearly, there is no discemible difference in convergence hetween the two versions of the flow solver for grid 1 , which is situated at the nose cone of the vehicle. The residual on yrid 5, which is situated at the rear of the velicle, converges for neitler method. This is due to the falce that the flow in this portion of the configuration is genuinely unslcildy, and no steidy state (\%cro residual) exists. Although a inisinatch exists between the residual evolutions of the two schemes, the general trend is similar, and there is no reason to helieve that the deferred version will be less stable than the original version. The mismatch can be removed by employing a subiteration strategy [13], which is a form of defect correction that is often used to reduce errors due to factorization and poor linearization. However, several random checks of some integrated physical quanticies (lift, drag, roll, pitch) for the X-38 from both the original and the deferred time-advancement strategies show differences of only a few tenths of a percent for all cases. Most likely, these differences are caused by the unsteady behavior behind the tail. Hence. it is not necessary to incur the additional cost of subiterations.

Having established that the deferred timestep method gives accurate results, we now turn our artention to its efficiency. Table 1 lists the runtime per timestep for three different configurations of eight processors. Evelyn and Piglet are each 8-processor Origin2000 systems located at NASA Ames, connected via a HIPPI channel. Denali is a 96-processor Origin2000 at Argonne, connected to Evelyn and Piglet via a DS3 Internet connection. To eliminate variability, all timings are the minimum over at least 15 rials.

\begin{tabular}{||c|c|c||c|c||}
\hline \hline \multicolumn{3}{|c||}{ \# Processors } & \multicolumn{2}{c||}{ secs/timestep } \\
\hline Evelyn & Piglet & Denali & Original & Deferred \\
\hline \hline 4 & 4 & & 16.2 & 12.7 \\
\hline 8 & & & 18.0 & \\
\hline & 4 & 4 & 21.4 & 21.3 \\
\hline
\end{tabular}

Table 1. Wall clock time (in secs) for each timestep of the CFD solver using the original and the deferred methods for three different configurations.

The first row of Table 1 shows that the deferred method does indeed hide at least some of the communication time between two supemodes connected by a rather highpertormance HIPPI channel. The second row shows that the application actually runs slower on a single 8-processor supernode than it does on two HIPPI-connected 4-processor supernodes (no difference between the original and the deferred methods). This is explained by the fact that the Origin2000 hardware does not support asynchronous message passing, but there are separate DMA engines on the: HIPPI boards that do suppor asynchronous communication tetween separate systems.

Finally. the third row shows no significant difference between the original and the deferred methods using the hincrnet connection. This result is expected becillse this contiguration deses not idlow asynchronous nessiging. To overconke this prohlem, we are currently investigalling lle use 
of dedicated nodes to serve as communication processors. This row of dara is included only to show the degradation in pertormance due to the relatively low-pertormance Intemet connection.

\section{Summary and future directions}

This paper described an experiment in which a largescale application in computational fluid dynamics (CFD) was adapted for efficient execution on the distributed environment of the Information Power Grid (IPG). The Globus metacomputing toolkit was used as the enabling software in this project. The CFD scheme uses structured overset grids and an enhanced version of the OVERFLOW code. The MPI-based OVERFLOW/Chimera application appears to be well-suited for a proof-of-concept demonstration of IPG/Globus technology. The application is very important to NASA, and will soon require resources exceeding what is available in any one tightly-coupled parallel system operated by NASA or its partners.

Load imbalance and communication overhead (given the smaller bandwidth and the larger latency due to the geographical separation of the computational resources used) werc idcntified as the main sources if parallel inefficiency. We anticipate alleviating the load imbalance problem by incorporating a "breakup" scheme to split large near-body grids, through better measurement of the loads associated with each grid, and by using a graph partitioner to more effectively assign grids to processors. Preliminary results showing that the application tolerates an additional lag of one timestep in boundary value interpolation suggests that the extra latency and reduced bandwidth involved in geographically-remote communication can be at least partially hidden. However, significant effort is still required to make the IPG a generally useful and a widely accessible environment for solving major computational problems.

\section{Acknowledgements}

The authors gratefully acknowledge the assistance of Andrew Wissink of MCAT, Inc., and Rober Meakin of Army/NASA AFDD for making the adaptive rectilinear: grid version of OVERFLOW available to us for the IPG experiments. The authors would also like to thank Terrence McGuinuess of San Diego Stare University for helping us examine the communication capabilities of our Origin2000 computers. This work was partially supported hy NASA under Contract Number NAS 2-14303 with MRJ Technology Solutions and under Contract Number NAS 2-13619 with Sterling Soliware.

\section{References}

[I] D. Bhatia, V. Burzevski, M. Camuseva, G. Fox, W. Furmenski. and G. Premchandran. Webtlow - a visual programming paradigm for Web/Java besed coarse grain distributed computing. Concurrency: Practice and Experience, 9:555-577, 1997.

[2] P. Buning, W. Chan, K. Renze, D. Sondak. I.-T. Chiu. J. Slotnick, R. Gomez, and D. Jespersen. Overflow user's manual, version 1.6au. NASA Ames Research Center, 1995.

[3] I. Foster, J. Geisler, W. Gropp, N. Karonis, E. Lusk, G. Thin vathukal, and S. Tuecke. Wide-area implementation of the Message Passing Interface. Parallel Computing. 24: 1735-1749, 1998.

[4] I. Foster. J. Geisler. W. Nickless. W. Smith. and S. Tuecke. Software infrastructure for the I-WAY high performance distributed computing experiment. In Sth IEEE Symp. on High Performance Distribused Compuring. pages 562-571, 1997.

[5] I. Foster and C. Kesselman. Globus: A metacomputing infrastnucture toolkit. Intemational Sournal of Supercomputer Applications, 11:115-128, 1997.

[6] I. Foster and C. Kesselman, editors. The Grid: Blueprint for a New Computing Infrastructure. Morgan Kaufmann, 1999.

[7] A. Geist, A. Beguelin, J. Dongarm, W. Jiang. R. Manchek, and V. Sunderam. PVM: Parallel Virtual Machine. MIT Press, 1994.

[8] A. Grimshaw. W. Wulf, and the Legion team. The Legion vision of a worldwide virtual computer. Communcations of the ACM, 40:39-45, 1997.

[9] W. Gropp, E. Lusk, N. Doss, and A. Skjellum. A highperformance, portable implementation of the MPI Message Passing Interface Standard. Parallel Computing, 22:789828, 1996.

[10] W. Jobnston. D. Gannon. W. Nitzberg, and W. V. Dalsem. Information Power Grid implementation plan. Working Draft, NASA Ames Research Center. 1998.

[11] G. Karypis and V. Kumar. A fast and high quality multilevel scheme for partitioning irregular graphs. Department of Computer Science Tech. Rep. 95-035, University of Minnesola, 1995.

[12] R. Meakin. On adaptive refinement and overset structured grids. In 13 th AIAA Computational Fluid Dynamics Conf. AlAA-97-1858, 1997.

[13] T. Pulliam. Time accuracy and the use of implicit methods. In 1 ith AIAA Computational Fluid Dynamics Conf., ALAA93-3360. 1993

[14] J. Sleger, F. Dougherty, and J. Benek. A Chimera grid scheme. ASME FED, 5, 1983.

[15] J. Waldo. Jini architecture overview. Sun Microsystems, 1998: http://java.sun.con/products/jini/whitepapers.

[16] A. Wissink and R. Meakin. Computational fuid dynamics with adaptive overset grids on parallel and distributed computer platforms. In Intl. Conf. on Parallel and Distributed Processing Techriques and Applications, pages 1628-1634. 1998. 